\title{
Constraining the nature of the accreting binary in CXOGBS J174623.5-310550
}

\author{
M. A. P. Torres, ${ }^{1,2,3 \star}$ S. Repetto ${ }^{\oplus},{ }^{4,5}$ T. Wevers, ${ }^{3,5,6}$ M. Heida ${ }^{\oplus},{ }^{7}$ P. G. Jonker,,${ }^{3,5}$ \\ R. I. Hynes, ${ }^{8}$ G. Nelemans, ${ }^{5,9}$ Z. Kostrzewa-Rutkowska, ${ }^{3,5}$ L. Wyrzykowski, ${ }^{10}$ \\ C. T. Britt, ${ }^{11}$ C. O. Heinke ${ }^{\oplus},{ }^{12,13}$ J. Casares, ${ }^{1,2}$ C. B. Johnson, ${ }^{8}$ T. J. Maccarone ${ }^{11}$ \\ and D. T. H. Steeghs ${ }^{14}$
}

${ }^{1}$ Instituto de Astrofísica de Canarias, Vía Láctea, La Laguna, E-38205 Santa Cruz de Tenerife, Spain

${ }^{2}$ Departamento de Astrofísica, Universidad de La Laguna, E-38206 Santa Cruz de Tenerife, Spain

${ }^{3}$ SRON, Netherlands Institute for Space Research, Sorbonnelaan 2, NL-3584 CA Utrecht, the Netherlands

${ }^{4}$ Physics Department, Technion - Israel Institute of Technology, Haifa 32000, Israel

${ }^{5}$ Department of Astrophysics/IMAPP, Radboud University, PO Box 9010, NL-6500 GL Nijmegen, the Netherlands

${ }^{6}$ Institute of Astronomy, Madingley Road, Cambridge CB3 OHA, UK

${ }^{7}$ Space Radiation Laboratory, California Institute of Technology, Pasadena, CA 91125, USA

${ }^{8}$ Department of Physics and Astronomy, Louisiana State University, Baton Rouge, LA 70803, USA

${ }^{9}$ Institute for Astronomy, KU Leuven, Celestijnenlaan 200D, B-3001 Leuven, Belgium

${ }^{10}$ Warsaw University Astronomical Observatory, Al. Ujazdowskie 4, PL-00-478 Warszawa, Poland

${ }^{11}$ Department of Physics \& Astronomy, Texas Tech University, Box 41051, Lubbock, TX 79409-1051, USA

${ }^{12}$ Department of Physics, University of Alberta, CCIS 4-183, Edmonton, AB T6G 2E1, Canada

${ }^{13}$ Max Planck Institute for Radio Astronomy, Auf dem Hugel 69, D-53121 Bonn, Germany

${ }^{14}$ Department of Physics, University of Warwick, Coventry CV4 7AL, UK

Accepted 2019 May 16. Received 2019 April 23; in original form 2018 November 5

\begin{abstract}
We report optical and infrared observations of the X-ray source CXOGBS J174623.5-310550. This Galactic object was identified as a potential quiescent low-mass X-ray binary accreting from an M-type donor on the basis of optical spectroscopy and the broad $\mathrm{H} \alpha$ emission line. The analysis of X-shooter spectroscopy covering three consecutive nights supports an M2/3-type spectral classification. Neither radial velocity variations nor rotational broadening is detected in the photospheric lines. No periodic variability is found in $I$ - and $r^{\prime}$-band light curves. We derive $r^{\prime}=20.8, I=19.2$, and $K_{\mathrm{s}} \approx 16.6$ for the optical and infrared counterparts with the M-type star contributing $\approx 90$ per cent to the $I$-band light. We estimate its distance to be $1.3-$ $1.8 \mathrm{kpc}$. The lack of radial velocity variations implies that the M-type star is not the donor star in the X-ray binary. This could be an interloper or the outer body in a hierarchical triple. We constrain the accreting binary to be a $\lesssim 2.2 \mathrm{~h}$ orbital period eclipsing cataclysmic variable or a low-mass X-ray binary lying in the foreground of the Galactic bulge.
\end{abstract}

Key words: binaries: close - stars: individual: CXOGBS J174623.5-310550 -X-rays: binaries.

\section{INTRODUCTION}

Interacting binaries containing compact objects provide a means to study the evolution of stars in binaries, and in particular the formation of their most compact remnants: neutron stars or black holes (BHs). The current Galactic population of accreting stellar-mass BHs amounts to 18 objects with a dynamical mass measurement (Casares \& Jonker 2014; Corral-Santana et al. 2016). Increasing

^E-mail: mapt@iac.es the sample of accreting Galactic BHs is of great importance for three main reasons. First, different supernova models predict a different BH mass distribution (Fryer \& Kalogera 2001; Belczynski et al. 2012; Fryer et al. 2012; Ugliano et al. 2012). Therefore, an unbiased determination of the $\mathrm{BH}$ mass distribution can be used to constrain supernova models. Secondly, increasing the sample of BHs with measured space velocities would help in the long debate on the type of natal kicks received by $\mathrm{BHs}$ at formation, which again provides input to supernova and binary evolution models (Jonker \& Nelemans 2004; Miller-Jones 2014; Repetto \& Nelemans 2015; Repetto, Igoshev \& Nelemans 2017). Thirdly, the comparison 
between the observed number of accreting $\mathrm{BH}$ binaries and the predicted number from population synthesis models would help in unraveling the physics involved in the formation and evolution of these sources (Romani 1992; Portegies Zwart, Verbunt \& Ergma 1997; Kalogera \& Webbink 1998; Pfahl, Rappaport \& Podsiadlowski 2003; Belczynski \& Taam 2004; Kiel \& Hurley 2006; Yungelson et al. 2006).

In an attempt to enlarge the sample of compact X-ray binaries and thus address the above questions, the Chandra Galactic Bulge Survey (GBS) imaged in X-rays a $12 \mathrm{deg}^{2}$ area towards the bulge with a limiting sensitivity set to maximize the number of detected quiescent low-mass X-ray binaries (LMXBs) over cataclysmic variables (CVs; see Jonker et al. 2011 and Jonker et al. 2014 for complete details on the GBS design). The X-ray survey has been complemented with dedicated multifrequency and variability studies, e.g. Maccarone et al. (2012), Hynes et al. (2012), Udalski et al. (2012), Greiss et al. (2014), Britt et al. (2014), Wevers et al. (2016a), and Wevers et al. (2017). Such studies are facilitating the identification of multiband counterparts to the $1640 \mathrm{X}$-ray sources found in the GBS. These counterparts are being classified on the basis of their spectroscopic and photometric properties (see Maccarone et al. 2012; Britt et al. 2013; Torres et al. 2014). Further photometry and spectroscopy is performed for candidate accreting binaries in order to establish the nature of their accretors (e.g. Ratti et al. 2013; Wevers et al. 2016b; Johnson et al. 2017). In this paper we present the follow-up observations of CXOGBS J174623.5-310550 (hereafter referred to as CX1004). CX1004 was detected with three $0.3-8 \mathrm{keV}$ counts during the Chandra GBS survey (Jonker et al. 2011), implying a $0.5-10 \mathrm{keV}$ X-ray luminosity of $L_{x}(d) \sim 2 \times 10^{31} \times\left(\frac{d}{1.0 \mathrm{kpc}}\right)^{2} \mathrm{erg} \mathrm{s}^{-1}$. Its optical counterpart was found at $r^{\prime}=20.75 \pm 0.02$ and $i^{\prime}=19.35 \pm 0.01$ in observations taken with the 4-m Victor M. Blanco telescope camera Mosaic-II in 2006 June (Wevers et al. 2016a). A subsequent Mosaic-II $r^{\prime}$-band light curve obtained during 2010 July did not show any significant photometric variability on a time-span of eight days (Britt et al. 2014; Torres et al. 2014). Optical spectroscopy taken with the VIsible Multi-Object Spectrograph (VIMOS) at the Very Large Telescope (VLT) and with the Gemini Multi-Object Spectrograph (GMOS) were presented in Torres et al. (2014) and Wu et al. (2015). The data showed absorption features consistent with those of an early M-type star and a broad double-peaked $\mathrm{H} \alpha$ emission line with $2120-2500 \mathrm{~km} \mathrm{~s}^{-1}$ full width at half-maximum (FWHM), flagging CX1004 as an accreting binary, either a low accretion rate highinclination CV or a quiescent LMXB.

This paper is organized as follows: the observations and data resuction steps are detailed in Section 2, Table 1 and Section 3. In Section 4 the optical spectra and the optical/infrared photometry are analysed. In Section 5 a discussion of the results is presented. Our conclusions are drawn in Section 6.

\section{SPECTROSCOPIC DATA}

Time-resolved spectroscopy of CX1004 was obtained using the medium resolution X-shooter echelle spectrograph (Vernet et al. 2011) mounted at the 8.2-m ESO Unit 2 VLT. The observations were obtained under program 088.D-0096(A). X-shooter provides spectra covering a large wavelength range of 3000-24 $800 \AA$, split into three spectroscopic arms: UVB, VIS, and NIR. For our analysis we focus on the VIS and UVB data, which cover the range $\lambda \lambda 3000$ 10240 with a dispersion of $\sim 0.2 \AA$ pixel $^{-1}$ in both arms. The NIRarm data were not used due to their lower signal-to-noise ratio
(SNR) and the light contamination from a nearby field star. The observations were taken with a slit width of 0 ".9 in the VIS arm and of 1 ". 0 in the UVB arm which delivered a resolving power of $\simeq 8800$ and $\simeq 5100$, respectively. We executed observing blocks consisting of an ABBA nodding sequence, with integration times for each spectrum of $900 \mathrm{~s}$ (VIS) and $877 \mathrm{~s}$ (UVB). To reduce systematic effects due to possible excursions of the target position with respect to the slit centre, we re-acquired CX1004 at the start of each one-hour-long observing block. We also observed with the same set-up and in nodding mode three red dwarfs that we will use for the analysis presented in Section 4.1: GJ 9592, GJ 465, and GJ 402 with spectral types M1, M2, and M4, respectively.

Six, eight, and six spectra were collected on 2012 March 1 from airmass 1.6 to 1.2 , on 2012 March 2 from airmass 1.9 to 1.2 , and on 2012 March 3 from airmass 1.6 to 1.2, respectively. From the FWHM of the collapsed spatial profile of the source spectrum at spectral positions close to $\lambda 6300$ we measure a mean image quality ranging from $1^{\prime \prime} .7$ to $1^{\prime \prime} .4,3$.'4 to $2^{\prime \prime} .0$, and $3^{\prime \prime} .2$ to $1^{\prime \prime} .5$ for the first, second, and third night, respectively. A similar FWHM was found at positions near $\lambda 9000$. Therefore, the VIS and UVB data were obtained in slit-limited conditions. On the other hand, because of the faintness of the source and the poor seeing during the observations, the individual spectra have low SNR: $\sim 2-5$ near $\mathrm{H} \alpha$ and $\sim 10$ in regions covering the $\mathrm{Ca}$ triplet.

We reduced each individual $900 \mathrm{~s}$ VIS and each 877 s UVB frame in order to optimize the time resolution. We processed the data using ESOREX, a software package delivered within the $\mathrm{X}$ shooter pipeline (Modigliani et al. 2010). In this way, the data were bias and flat-field corrected, the echelle orders were merged and rectified, and the spectra wavelength calibrated. After several tests to investigate how to maximize the signal-to-noise of the extracted one-dimensional spectra, we performed the extraction of each twodimensional spectrum with IRAF using an extraction aperture with size equal to the FWHM measured from the spatial profile of the spectrum in question. The resulting extracted spectra of CX1004 and the spectral-type templates were subsequently imported in MOLLY, rebinned to a uniform pixel scale. We checked the zero-point of the wavelength calibration of our spectra measuring the velocity shift of the sky emission lines [O I] $\lambda \lambda 6300.304,8310.719$ (Osterbrock et al. 1996). We used these shifts to correct for the zero-point deviations in the spectral regions covering $\mathrm{H} \alpha$ and the Ca II infrared triplet. The median offsets in the $\lambda 6300$ and $\lambda 8311$ sky lines were $\leq 3.6$ and $\leq 4.3 \mathrm{~km} \mathrm{~s}^{-1}$ in amplitude, respectively. No suitable sky emission lines were available in the spectral range covered by the UVB arm, thereby a zero-point correction was not possible. Finally, the spectra were corrected for the motion of the Earth.

\section{PHOTOMETRIC DATA}

\subsection{GBS optical point source catalogue}

CX1004 is found in the GBS optical point source catalogue (Wevers et al. 2016a) which consists of optical photometry obtained using the Mosaic-II imager on the 4-m Victor M. Blanco telescope at CTIO. With eight CCDs, the instrument covered a $36^{\prime} \times 36^{\prime}$ field of view (FOV) with a plate scale of 0.27 pixel $^{-1}$. The photometry was taken between 2016 June 21 and 2016 June 29. The GBS area was covered in the $r^{\prime}, i^{\prime}$, and $\mathrm{H} \alpha$ filters, with exposure times of 120 , 180 , and $480 \mathrm{~s}$, respectively. The data reduction steps, as well as the photometric and astrometric calibration, are described in detail in Wevers et al. (2016a). This catalogue is our photometric and astrometic reference or the calibration of the new data presented 
in this paper (Sections 3.4, 3.5) and for the recalibration of other, previously published photometry (Section 4.3).

\subsection{OGLE}

CX1004 is listed as object ID 18319 in the bulge field BLG659.29 monitored during the fourth phase of the Optical Gravitational Lensing Experiment (OGLE-IV). Between 2010 April 23 and 2011 March 19 a total of $60 \mathrm{I}$-band data points were collected with the 1.3-m Warsaw telescope located at Las Campanas observatory under a $\approx 1^{\prime \prime} .3$ seeing sampled with a plate scale of 0.26 pixel $^{-1}$. Photometry was obtained using the difference imaging technique tied to the OGLE data (see Udalski, Szymański \& Szymański 2015 for a detailed overview of the OGLE-IV survey).

\section{$3.3 \mathrm{VVV}$}

Archival infrared images from the VISTA Variables in the Via Lactea Survey (VVV, Minniti et al. 2010) were inspected to search for the infrared counterpart to CX1004. The survey data were obtained with the 4-m VISTA telescope at Paranal Observatory that used VIRCAM, a camera with a $1.1 \times 1.5 \mathrm{deg}^{2}$ FOV and a plate scale of 0.34 pixel $^{-1}$. In our study we used data acquired in the $K_{s}$-band during 2012 August 15 and 2013 March 23 with total time on source of $48 \mathrm{~s}$ and an image quality better than 0.8 .

\subsection{DECam}

As a continuation of the GBS variability survey published in Britt et al. (2014), time-resolved photometry of the field containing CX1004 was obtained on 2013 June 10-11 with the Dark Energy CAMera (DECam; DePoy et al. 2008) on the 4-m Victor M. Blanco telescope at CTIO. DECam uses a mosaic of $62 \mathrm{CDD}$ each with $2 \mathrm{k} \times 4 \mathrm{k}$ pixels to cover a $2.2 \mathrm{deg}^{2}$ FOV with a plate scale of $0^{\prime \prime} 27$ pixel $^{-1}$. A total of $109 r^{\prime}$-band images were taken with an exposure time of $90 \mathrm{~s}$ each. The seeing was between 0.8 and 2.5 over both nights. The images were reduced with the NOAO DECam pipeline and astrometry was performed using the pipeline WCS on the reprojected images. Instrumental magnitudes were extracted using point spread function (PSF) photometry with the DAOPHOT task in IRAF. Finally, the photometric calibration was performed against the GBS point source catalogue (Section 3.1).

\subsection{FORS2}

Single $B$ - and $I$-band images of the field containing CX1004 were obtained with the FOcal Reducer and low dispersion Spectrograph 2 (FORS2; Appenzeller et al. 1998) mounted on the 8.2-m ESO Unit 1 VLT. The observations were obtained under program 095.D0973(A) during 2015 June 12. The instrument was used with the standard resolution collimator $\left(6^{\prime} .8 \times 6^{\prime} .8 \mathrm{FOV}\right)$ and the mosaic of two $2 \times 4 \mathrm{k}$ MIT CCDs. The CCDs were binned $2 \times 2$ providing a plate scale of 0.25 pixel $^{-1}$. The integration times were 300 and $30 \mathrm{~s}$ for the images taken with the $B$ - and $I$-Bessel filters, respectively. The image quality was 0.99 ( $B$ band) and 0.75 FWHM ( $I$ band). The data were bias subtracted and flat-field corrected using standard tasks in IRAF. The computed astrometric solution for the images had an r.m.s of $\approx 0$ '. 162 . Then we used IRAF tools to shift the images to the same reference frame using the centroids of 103 point sources in the images.

\section{DATA ANALYSIS AND RESULTS}

\subsection{Determining the radial velocities, spectral type, and rotational broadening of the M-type star}

We measure the radial velocities (RVs) of the candidate counterpart, cross-correlating its X-shooter spectra with those of a template star (Tonry \& Davis 1979). Prior to the cross-correlation, target and template spectra were resampled into a common logarithmic wavelength scale. Next, all spectra were normalized over the range $\lambda \lambda 8010-8810$ by dividing them with the result of a third-order spline function fit to the continuum obtained while masking strong spectral features. The normalized wavelength range contains the resolved Ca II infrared triplet that, in contrast to other photospheric lines, is detected in most of the individual spectra despite their low SNR. The Na I doublet is also evident, but we choose not to include it in the analysis since it is contaminated by telluric lines. All computed cross-correlation functions showed a significant peak, with best results achieved when using only the wavelength intervals covering the sharp Ca II triplet lines. The RV values provided in this paper were obtained by cross-correlating the target data against the M2-type template, which best matches the spectral type for CX1004 (see below). We corrected the resulting velocities for the intrinsic $51.17 \mathrm{~km} \mathrm{~s}^{-1}$ systemic RV of the M2 dwarf, which is accurate to approximately $0.1 \mathrm{~km} \mathrm{~s}^{-1}$ (Nidever et al. 2002). The resulting RVs do not show any significant variations during the three nights of observations. Their nightly means and standard deviations are: $-23 \pm 3,-25 \pm 5$, and $-24 \pm 3 \mathrm{~km} \mathrm{~s}^{-1}$ for 1,2 , and 3 of March, respectively.

Torres et al. (2014) supported an early M-type classification for the optical counterpart to $\mathrm{CX} 1004$ on the basis of the presence of prominent $\mathrm{TiO} \alpha, \beta, \gamma, \gamma^{\prime}$ band systems and the lack of the TiO $\delta, \epsilon$ band systems redward of $8000 \AA$ in VIMOS spectra. The $\mathrm{X}$-shooter spectra confirm these results. In particular, there is no evidence for $\mathrm{TiO}$ molecular bands in the range $\lambda \lambda 8200-8800$ (see Fig. 1). Molecular bands start to be evident in this region for spectral types later than M3 (see e.g. Jones, Alloin \& Jones 1984; Zhong et al. 2015). To verify our visual classification that does not account for the possible contribution from an accretion flow to the optical continuum, we apply the optimal subtraction method described in Marsh, Robinson \& Wood (1994). This method allows to determine the spectral type of the stellar component, its fractional contribution to the total light, and the rotational broadening of the photospheric lines. We focused this analysis on the Ca II infrared triplet region where the SNR is higher.

First, the normalized target spectra were velocity-shifted to the rest frame of the template star by subtracting the RVs obtained from the cross-correlation with the template. Next, the velocityshifted spectra were averaged, with different weights to maximize the SNR of the resulting sum. The spectral templates were then broadened from 0 to $100 \mathrm{~km} \mathrm{~s}^{-1}$ in steps of $2 \mathrm{~km} \mathrm{~s}^{-1}$ through convolution with the rotational profile of Gray (1992) adopting a limb-darkening coefficient of 0.75 . Each broadened version of the template spectrum was multiplied by a varying factor $f$ (representing the fractional contribution of light from the template star) and next subtracted from the CX1004 average spectrum. Then a $\chi^{2}$ test on the residuals was performed to find the optimal value of $f$ by minimizing the $\chi^{2}$ between the residual of the subtraction and a smoothed version of itself. We took the average of the $\chi^{2}$ and $f$ values obtained by smoothing the residual using a Gaussian with FWHM from 25 to $100 \AA$. For each template, we are then able to produce a $\chi^{2}$-curve as a function of the applied broadening, whose 


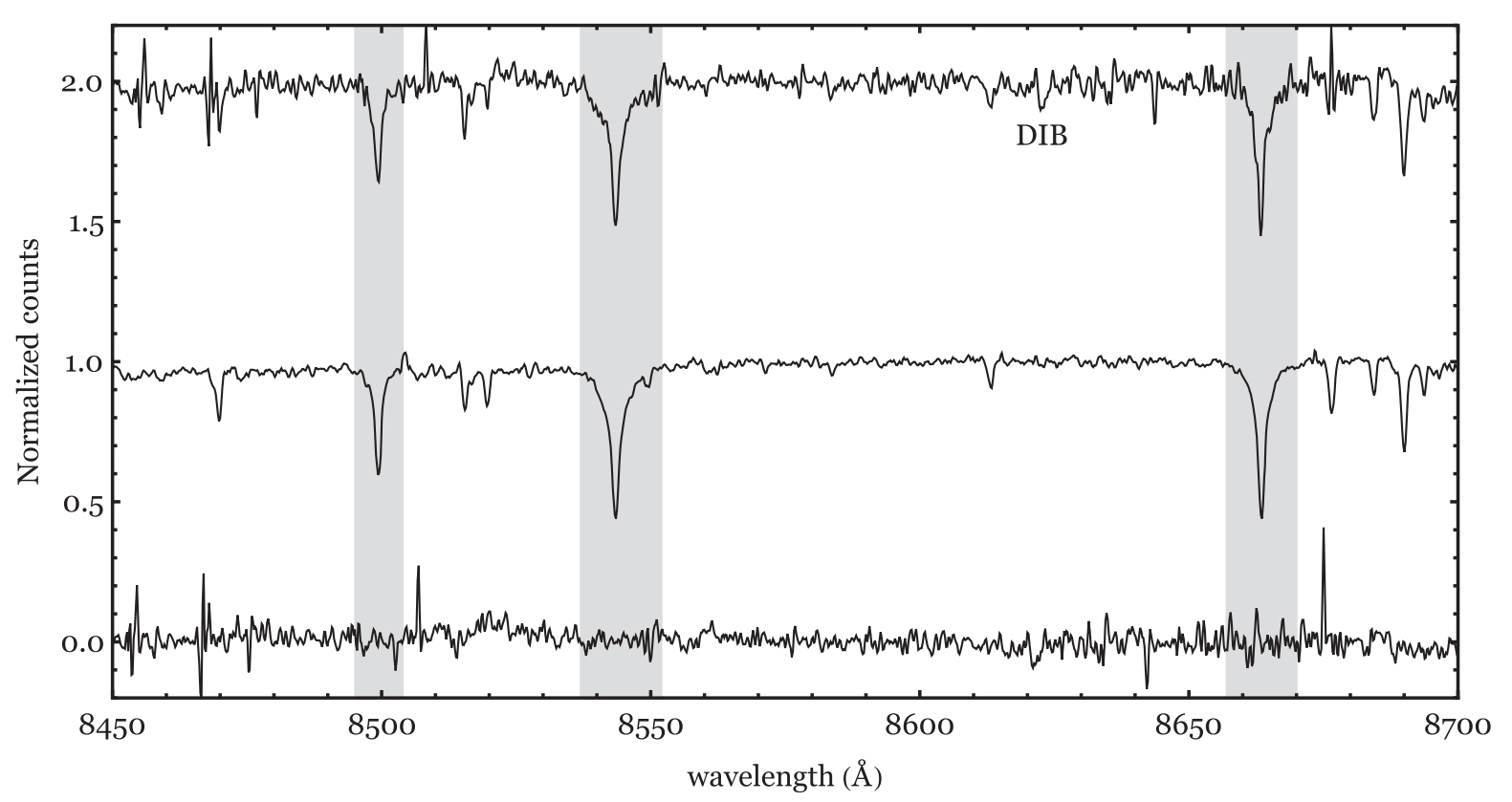

Figure 1. From top to bottom: the normalized velocity-corrected average spectrum of CX1004, the M2 template, and the residuals after optimal subtraction. Arbitrary vertical offsets have been applied to the spectra for the sake of clarity. The shaded areas mark the Ca II triplet absorption lines. The $\lambda 8621$ DIB is also indicated.

minimum provides $V \sin i$ and $f$. The $V \sin i$ errors were obtained following the bootstrapping approach outlined in Steeghs \& Jonker (2007). We find that only in the case of the M4 V template the $\chi^{2}$ curve is non-monotonic with a single minimum, whereas for the M1 and M2 dwarf template, the $\chi^{2}$-curve is increasing monotonically with $V \sin i$. From the $\chi^{2}$-values (see Table 2), we derive that the M2 dwarf provides the best fit to the averaged spectrum, while the M4 template yields the highest $\chi^{2}$ and it has a non-physical value for $f$. Therefore, we conclude the M2 template best matches the spectrum of CX1004, although we cannot exclude a M3 spectral type for the star. In Fig. 1 we show the average CX1004 spectrum (top), the M2 template (middle), and the residual after the optimal subtraction (bottom). Both the averaged and residual spectrum show a diffuse interstellar band (DIB) at $\lambda 8621$. We will use its equivalent width (EW) of $0.19 \pm 0.06 \AA$ to estimate the reddening in Section 4.4. Our analysis shows that the M2 template contributes $\approx 90$ per cent to the total $I$-band flux. The results also imply a low rotational broadening for the photospheric lines $V \sin i \lesssim 35 \mathrm{~km} \mathrm{~s}^{-1}$, an upper limit set by the spectral resolution measured in the $\mathrm{Ca}$ II triplet region. In the remainder of this paper we will refer to the M2/3 star associated to the optical counterpart as the M2 star or M2 companion.

\subsection{The properties of detected emission lines}

$\mathrm{H} \alpha$ is the only emission line present in the X-shooter VIS part of the spectra. Its double-peaked morphology is apparent in the individual spectra with the highest SNR. $\mathrm{H} \beta$ and $\mathrm{H} \gamma$ emission lines are only detected after averaging the UVB data (see Fig. 2). To characterize the $\mathrm{H} \alpha$ emission line profile, we first normalized the spectra by fitting the continuum adjacent to the line with a low-order spline function. Next, we produced nightly average spectra to increase the SNR. In this process, we discarded data being consistent with noise in the wavelength interval of interest - five and one spectra obtained in the second and third night, respectively. The resulting spectrum is shown in Fig. 2 together with the normalized M2 template spectrum.
By comparing them, it becomes evident that the two more prominent narrow absorption features in the double-peaked $\mathrm{H} \alpha$ line are also present in the stellar template. We identify them as photospheric $\mathrm{H} \alpha$ and $\mathrm{Ca}$ I $\lambda 6573$ that originate in the M2 star. Thus, the $\mathrm{X}$ shooter resolving power allows us to exclude the possibility that the deep narrow $\mathrm{H} \alpha$ absorption is due to an inclination effect on the shape of the emission profile. Such effect is frequently seen in eclipsing CVs and also in high-inclination BH LMXBs (e.g. Marsh, Horne \& Shipman 1987, Torres et al. 2015). In what follows, we will mask this absorption feature when fitting the average double-peaked profiles.

Single and 2-Gaussian profiles were fit to the emission line to measure its FWHM and the velocity shift of the blue $\left(V_{\mathrm{b}}\right)$ and red $\left(V_{\mathrm{r}}\right)$ peaks with respect to the line's rest wavelength. The $\left(V_{\mathrm{r}}+\right.$ $\left.V_{\mathrm{b}}\right) / 2$ value provides the centroid $\mathrm{RV}$ with respect to the line rest wavelength while $\left|V_{\mathrm{r}}-V_{\mathrm{b}}\right|$ yields the peak-to-peak separation, $\Delta V^{p p}$. The results from the fits are given in Table 3 together with the EW of the lines. The uncertainties in the EWs were estimated from the scatter in the values obtained using different wavelength intervals to place the local continuum level. We deem the difference between the RV values obtained for the per night average $\mathrm{X}$-shooter spectra to be due to noise on the line structure rather than to RV changes in the line centroid. The first night of observations has in general the highest SNR spectra thus dominating the resulting weighted average of the 14 line profiles. Note that fits performed without masking the absorption component from the M2 star, yield larger FWHMs (by $\sim 100 \mathrm{~km} \mathrm{~s}^{-1}$ ) while the $\Delta V^{p p}$ and DP values increase by $\lesssim 2$ per cent. The EW measured for $\mathrm{H} \alpha$ are low compared to those observed in quiescent CVs and LMXBs. This is solely due to the strong contribution of the M2 star to the optical continuum. While the per night average EW from the X-shooter data are the same within the errors, they are 30 per cent lower than found from the GMOS observations indicating long-term variability of the line strength. We also normalized the wavelength interval containing $\mathrm{H} \beta$ and performed single and 2-Gaussian model fits for the average profile resulting from combining the three nights of 
Table 1. Photometric and spectroscopic data. The Mosaic-II, VIMOS, and GMOS data sets reanalysed in this work are published first in Britt et al. (2014), Torres et al. (2014), and Wu et al. (2015), respectively.

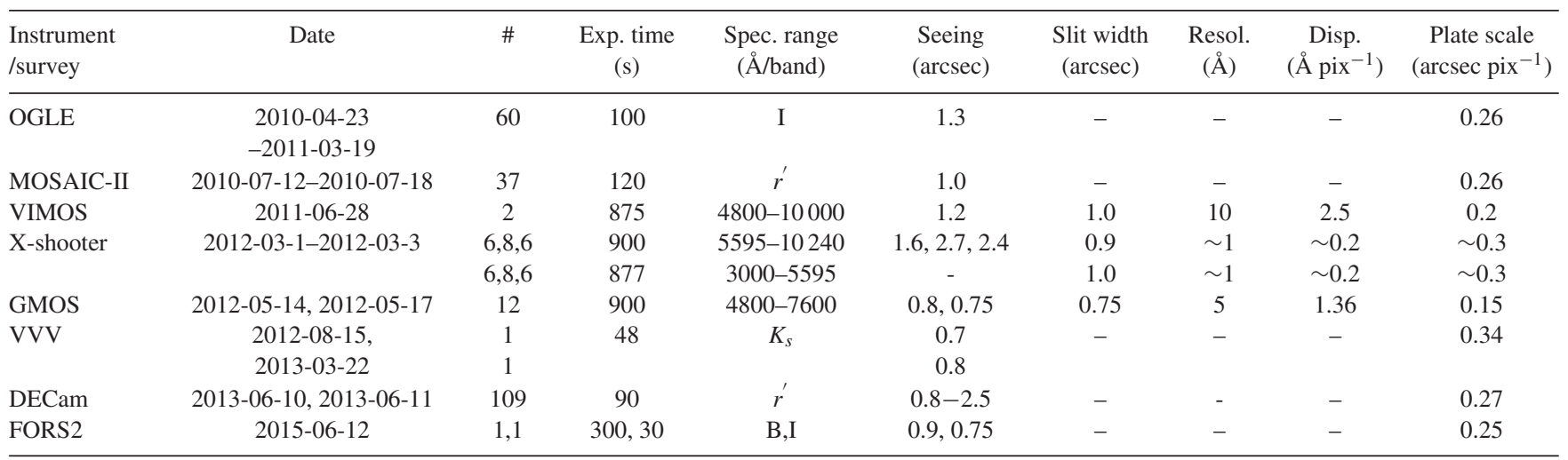

Table 2. Spectral classification and rotational broadening. d.o.f. $=$ degrees of freedom.

\begin{tabular}{lcccc}
\hline Template & $\begin{array}{c}\text { Spectral } \\
\text { type }\end{array}$ & $\begin{array}{c}V \sin i \\
\left(\mathrm{~km} \mathrm{~s}^{-1}\right)\end{array}$ & $f$ & $\begin{array}{c}\chi_{\min }^{2} / \text { d.o.f } \\
(\text { d.o.f } \approx 160)\end{array}$ \\
\hline GJ 9592 & M1 V & - & $1.00 \pm 0.05$ & 8.4 \\
GJ 465 & M2 V & - & $0.90 \pm 0.01$ & 7.8 \\
GJ 402 & M4 V & $19 \pm 4$ & $1.20 \pm 0.08$ & 9.6 \\
\hline
\end{tabular}

data. We provide the results from these fits in Table 3. The $\mathrm{H} \beta$ EW measurement is very uncertain given that the line continuum is difficult to establish. Finally, $\mathrm{H} \gamma$ is marginally detected in the averaged data and reliable fits were not possible.

Using VIMOS spectroscopy, Torres et al. (2014) measured a significant RV of $-170 \pm 20 \mathrm{~km} \mathrm{~s}^{-1}$ in both the centroid of the $\mathrm{H} \alpha$ profile and photospheric absorption lines. This result is in contrast with the low RVs obtained from the X-shooter data. To search for any systematic effects that could alter the velocity determinations, we reanalysed the VIMOS spectra finding an RV consistent with that reported in Torres et al. (2014). The multislit mask of the VIMOS observations of CX1004 was designed to have the counterpart centred on a 1".0 width slit. We examined the acquisition images and the spatial profile for the spectrum to confirm if that was the case. We found that the source was offset towards the North-East direction. This positional offset from the centre of the slit will have introduced a significant RV offset. Furthermore, light from a field star 1".3 N-E from CX1004 fell inside the slit contaminating its spectrum. ${ }^{1}$ Since we cannot quantify the velocity offset with the available data, we deem the RVs from the VIMOS spectra unreliable.

An RV of $-15 \pm 20 \mathrm{~km} \mathrm{~s}^{-1}$ was measured from GMOS spectroscopy using a double-Gaussian fit to the averaged $\mathrm{H} \alpha$ profile (Wu et al. 2015). To refine this determination and extract information from individual profiles, we corrected the GMOS spectra for wavelength zero-point offsets using the [O I] $\lambda 6300.304$ sky emission line. We recalculated the line parameters for the weighted average data as done above for the X-shooter data. In this case, during the fits we applied a mask centred on the doublepeaked emission line with a width of the GMOS spectral resolution to exclude regions affected by the unresolved line component from the M2 star. The results are shown in Table 3. The individual profiles were fit with a single Gausian model without masking the $\mathrm{H} \alpha$

${ }^{1}$ A finding chart is available in Appendix B in Torres et al. (2014). absorption line from the M2 star. The fits delivered a mean FWHM of $2430 \mathrm{~km} \mathrm{~s}^{-1}$ with r.m.s $=140 \mathrm{~km} \mathrm{~s}^{-1}$, consistent with the value obtained from the same fitting procedure applied to the averaged profile $\left(2510 \pm 40 \mathrm{~km} \mathrm{~s}^{-1}\right)$. We also cross-correlated the individual profiles against a Gaussian function with $F W H M=2450 \mathrm{~km} \mathrm{~s}^{-1}$ and calculated using a 2-Gaussian fit function the RV of the $\mathrm{H} \alpha$ centroid on the best quality individual data. None of the methods delivered evidence for significant variations in the line parameters.

\subsection{Light-curve analysis and astrometric matching of multiband counterparts}

In Fig. 3 we present the long-term (2010 April-2011 March) Iband OGLE light curve for CX1004. The source has an average magnitude of $I_{\mathrm{OGLE}}=19.21$, with an r.m.s. of $0.08 \mathrm{mag}$ and (after rejecting photometric points with $\mathrm{SNR}<15) \Delta \mathrm{I}=\mathrm{I}_{\max }-\mathrm{I}_{\min }=$ 0.3 . The light curve of field stars with similar $I$-band brightness have comparable r.m.s. and photometric errors $(\sim 0.05$ mag on average). We therefore find no evidence for photometric variability in the OGLE data. The field star 1".3 to the North-East from CX1004 has an average magnitude $I=20.1$ and r.m.s. of 0.1 mag. In our search for long-term variability we calibrate the 2015 June FORS2 I-band PSF photometry of both CX1004 and the N-E field star (Section 3.5) using the $I$-band magnitudes of field stars in the OGLEIV optical source catalogue (Udalski et al. 2015). We obtain $I_{\mathrm{FORS} 2}=$ $19.21 \pm 0.02$ and $20.14 \pm 0.02$, for the counterpart of CX1004 and the nearby field star, respectively, fully consistent with the OGLE photometry.

We show in Fig. 4 the 2013 June 10/11 $r^{\prime}$-band DECam light curve of CX1004 and the N-E field star. After removing the photometric points with false (correlated) variability and following Wevers et al. (2016b), we calculated periodograms over a period ranging from minutes to days. We find the light curve of CX1004 to be aperiodic and we measure its average $r^{\prime}$ magnitude, r.m.s. scatter, and maximum brightness variation to be $20.80,0.02$, and $0.12 \mathrm{mag}$, respectively. For the field star we obtain a mean magnitude of 21.94 and an r.m.s. of 0.06 mag. In comparison, the GBS optical source catalogue (Wevers et al. 2016a) reports $r^{\prime}=20.75 \pm 0.02$ from the 2006 observations of CX1004. When recalibrated with respect to the same stars used for the DECam data and after rejecting points with SNR $<15$, the 2010 July 12-18 Mosaic-II light curve (Britt et al. 2014) has a mean $r^{\prime}=20.83$, an r.m.s. of 0.04 , and a $\Delta r^{\prime}=0.16$. The small discrepancy with respect to the 2006 Mosaic-II photometry is likely due to the fact that the N-E star was not resolved in the latter. Thus there is no evidence for intrinsic variability of the source. 


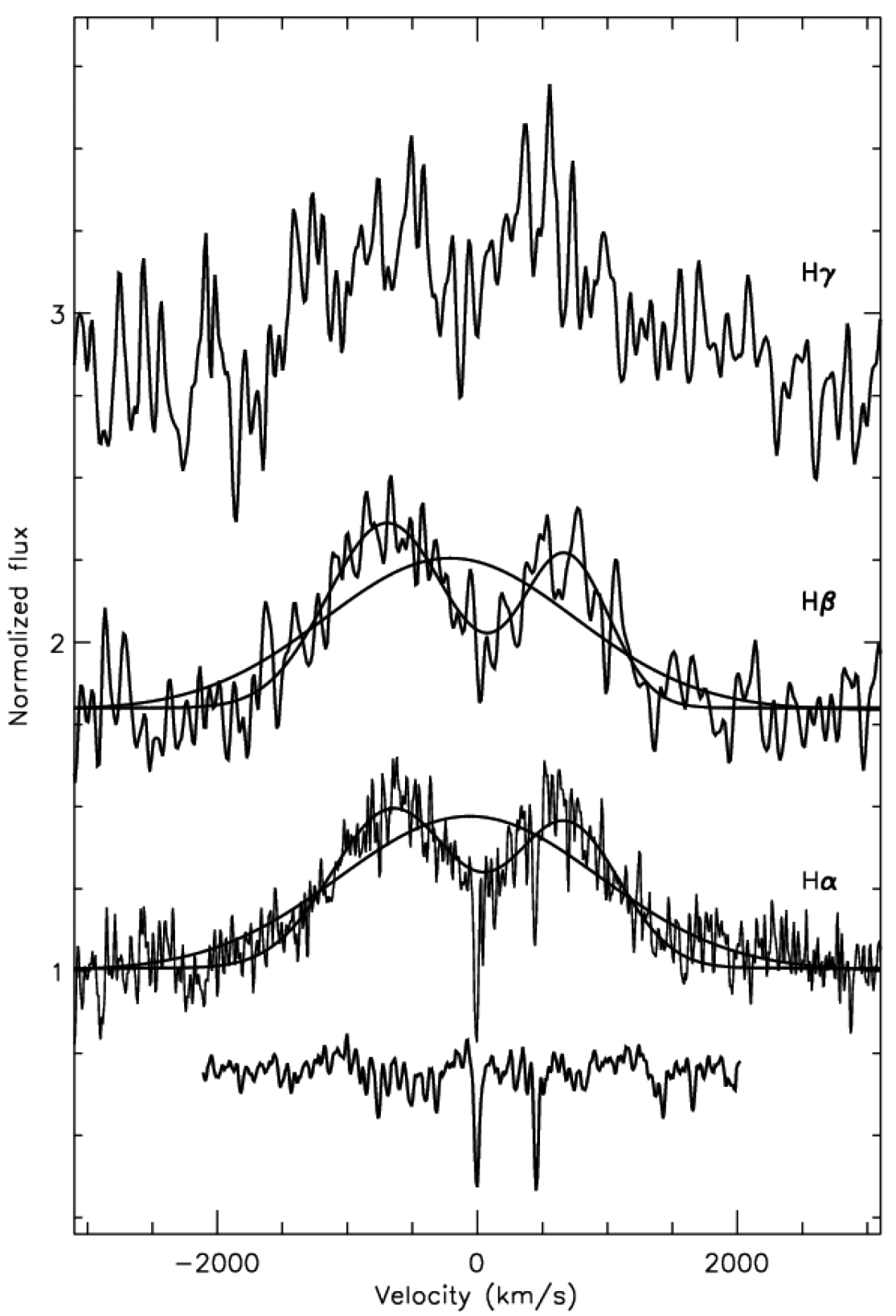

Figure 2. Average profile of the Balmer emission lines in the X-shooter spectra of CX1004. The spectra of the $\mathrm{H} \beta$ and $\mathrm{H} \gamma$ lines are smoothed with a 3-pixel-wide (FWHM) Gaussian. The single and double-Gaussian fits performed in Section 4.2 are overplotted. The bottom spectrum shows the M2 template where the strongest photospheric absorption lines correspond to $\mathrm{H} \alpha$ and $\mathrm{Ca}$ I $\lambda 6573$.

The lack of RV and photometric variations implies that the Mtype star is not associated with the $\mathrm{H} \alpha$-emitting source. This can mean that this star is an interloper unrelated to the X-ray binary or it might be the outer star in a hierarchical triple system. Thus we looked for potential positional offsets between multiband optical counterparts. For this search we analysed the aligned $B$ - and $I$-band FORS2 images of the field (Section 3.5). Aperture photometry was performed for 103 point sources within a $\approx 500$ arcsec radius from the target to derive their emission centroid in image coordinates. For each source, $\mathrm{X}$ and $\mathrm{Y}$ offsets were computed and added in quadrature after converting them into arcsec. The offset between the $I$ - and $B$-band counterparts to CX1004 is 0.'05. The fraction of the 103 sources with a larger offset is 6 per cent. We also performed PSF photometry on both images using DАОРНОT. We find no evidence for underlying sources or residuals in any of the images after subtracting the PSF model. These results imply that in the above photometric bands and at the time of the observations, the M-type star is the dominant source of light making it impossible to test the interloper scenario with our images.

Finally, by inspecting the archival VVV images, we detect an uncatalogued point-like source that matches the astrometric position of the optical counterpart to CX1004. To compute its instrumental $K K_{\mathrm{s}}$-band magnitude and that of other point-like objects in the field, we performed PSF photometry with DAOPHOT. Differential photometry with respect to a nearby and non-variable field star calibrated in VVV yields $K_{\mathrm{s}}=16.6 \pm 0.2$ for CX1004. The counterpart is not detected in the UKIRT Infrared Deep Sky Survey due to poor quality of the data for CX1004. In addition, we note that 
Table 3. Best-fitting parameters for the emission lines. The FWHMs are derived from a single Gaussian fit; while the line centroid RV and peak-to-peak separation $\left(\Delta V^{p p}\right)$ are obtained from a 2-Gaussian fit to the emission line. DP is the double-peak separation measured from the 2-Gaussian fit when both components are forced to have the same height and FWHM.

\begin{tabular}{|c|c|c|c|c|c|c|}
\hline $\begin{array}{l}\text { Line } \\
\text { instrument }\end{array}$ & $\begin{array}{c}\text { Night } \\
\text { average }\end{array}$ & $\begin{array}{c}\# \\
\text { Spectra }\end{array}$ & $\begin{array}{l}\text { FWHM } \\
\left(\mathrm{km} \mathrm{s}^{-1}\right)\end{array}$ & $\begin{array}{c}\mathrm{RV} \\
\left(\mathrm{km} \mathrm{s}^{-1}\right)\end{array}$ & $\begin{array}{l}\Delta V^{p p},(\mathrm{DP}) \\
\left(\mathrm{km} \mathrm{s}^{-1}\right)\end{array}$ & $\begin{array}{l}\text { EW } \\
(\AA)\end{array}$ \\
\hline \multicolumn{7}{|l|}{$\mathrm{H} \alpha \lambda 6562.760$} \\
\hline \multirow[t]{4}{*}{ X-shooter } & 2012 March 1 & 6 & $2330 \pm 20$ & $-10 \pm 5$ & $1310 \pm 10$ & $-22 \pm 2$ \\
\hline & 2012 March 2 & 3 & $2310 \pm 40$ & $-90 \pm 10$ & $1370 \pm 20$ & $-25 \pm 3$ \\
\hline & 2012 March 3 & 5 & $2460 \pm 30$ & $30 \pm 10$ & $1340 \pm 20$ & $-22 \pm 3$ \\
\hline & 2012 March 1-3 & 14 & $2360 \pm 20$ & $-10 \pm 5$ & $\begin{array}{r}1330 \pm 10 \\
(1320 \pm 10)\end{array}$ & $-22 \pm 2$ \\
\hline GMOS & 2012 May 17, 2012 May 14 & 12 & $2350 \pm 40$ & $-20 \pm 20$ & $\begin{array}{r}1350 \pm 40 \\
(1320 \pm 20)\end{array}$ & $-32 \pm 2$ \\
\hline \multicolumn{7}{|l|}{$\mathrm{H} \beta \lambda 4861.327$} \\
\hline X-shooter & 2012 March 1-3 & 17 & $2260 \pm 30$ & $-30 \pm 5$ & $1370 \pm 10$ & $\sim-20$ \\
\hline
\end{tabular}

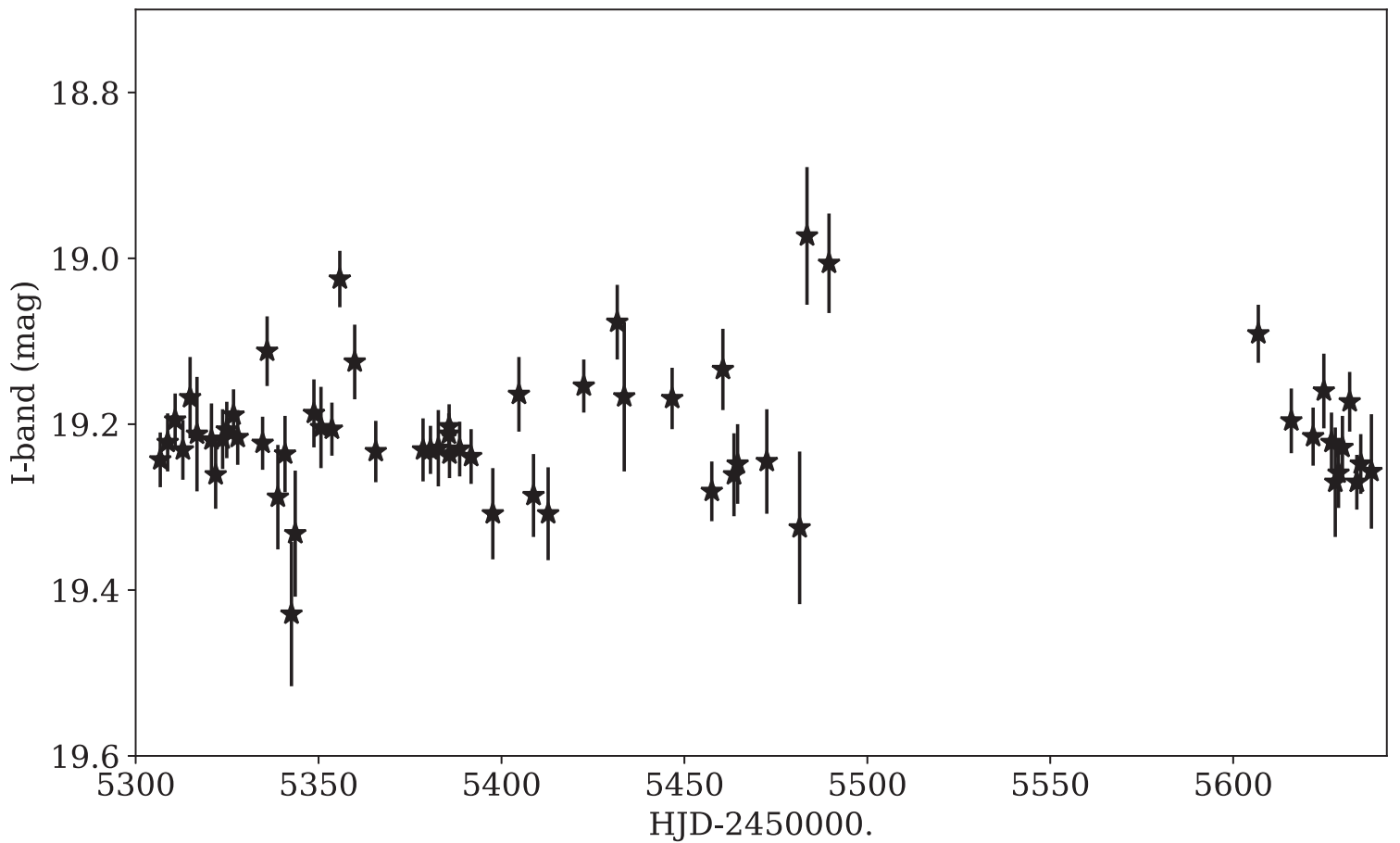

Figure 3. 2010 April 23-2011 March 19 OGLE I-band light curve of CX1004.

the $K_{\mathrm{s}} \approx 14.2$ candidate counterpart VVV J174623.57-310550.75 reported in Greiss et al. (2014) is a nearby field star unrelated to CX1004.

\subsection{Extinction and distance}

In this section we will employ the median values 1:1.85:13.44 for the ratios $A_{V}: A_{I}: A_{K_{s}}$ towards the bulge (Nataf et al. 2016), the ratio $\mathrm{A}_{\mathrm{K}_{\mathrm{s}}} / \mathrm{E}\left(\mathrm{J}-\mathrm{K}_{s}\right)=0.528$ (Nishiyama et al. 2009), and the three-dimensional $\mathrm{E}\left(\mathrm{J}-\mathrm{K}_{s}\right)$ map of the Galactic bulge by Schultheis et al. (2014). In addition, we will use the VISTA $\mathrm{K}_{s} \simeq \mathrm{K}+0.044$ transformation (Carpenter 2001; González-Fernández et al. 2018) to convert between VISTA and Bessell \& Brett photometric systems.

We derive an estimate of the extinction $\mathrm{A}_{\mathrm{K}_{\mathrm{S}}}$ using the EW of the DIB at $\lambda 8621$ which we measured in Section 4.1. The relation $\mathrm{A}_{\mathrm{K}_{\mathrm{s}}} \approx 0.691 \times \mathrm{EW}(\AA)$ for this DIB (Damineli et al. 2016) yields $\mathrm{A}_{\mathrm{K}_{\mathrm{s}}}=0.13 \pm 0.04$ from which we obtain $\mathrm{A}_{\mathrm{I}}=1.0 \pm 0.3$. We can also estimate the reddening from the observed $\left(\mathrm{I}-\mathrm{K}_{s}\right)$ colour of the source assuming that the 90 per cent of the $I$-band brightness (i.e. $I=19.3)$ and the total infrared emission $\left(\mathrm{K}_{s}=16.6 \pm 0.2\right)$ is due to the light from the M2-type star only. In this way we find an observed colour $\left(\mathrm{I}-\mathrm{K}_{s}\right)=2.7 \pm 0.2$, redder than the intrinsic colour $\left(\mathrm{I}-\mathrm{K}_{s}\right)_{0}$ of 2.02 (2.22) for M2(M3) dwarfs (Bessell 1991). Using $\mathrm{A}_{\mathrm{I}} / \mathrm{A}_{\mathrm{K}_{\mathrm{s}}}=7.26$ and the colour excess, we estimate $\mathrm{A}_{\mathrm{I}}=0.8 \pm 0.2$ consistent with the value calculated from the DIB. Taking the $I$-band absolute magnitude for an M2/M3 V star $\left(\mathrm{M}_{\mathrm{I}}=8.00 / 8.71\right.$; Bessell 1991 ) and adopting $A_{I}=0.9$, we find a distance of $\approx 1.8 / 1.3 \mathrm{kpc}$. We can also constrain the distance using the three-dimensional $\mathrm{E}(\mathrm{J}-$ $\mathrm{K}_{s}$ ) maps of the bulge: the map towards CX1004 delivers $\mathrm{E}\left(\mathrm{J}-\mathrm{K}_{s}\right)$ $=0.21$ and 0.31 (i.e. $\mathrm{A}_{\mathrm{I}}=0.8$ and 1.2) for a distance of 1.0 and $1.5 \mathrm{kpc}$. This is in line with the values found above for the distance which in turn supports a main-sequence luminosity class for the M2-type star.

Given that the M2-type star is not the donor star in CX1004, we derive $I \gtrsim 21.7$ for the accreting $X$-ray binary when accounting for the fact that this contributes $\lesssim 10$ per cent to the 19.2 mag $I$ - 


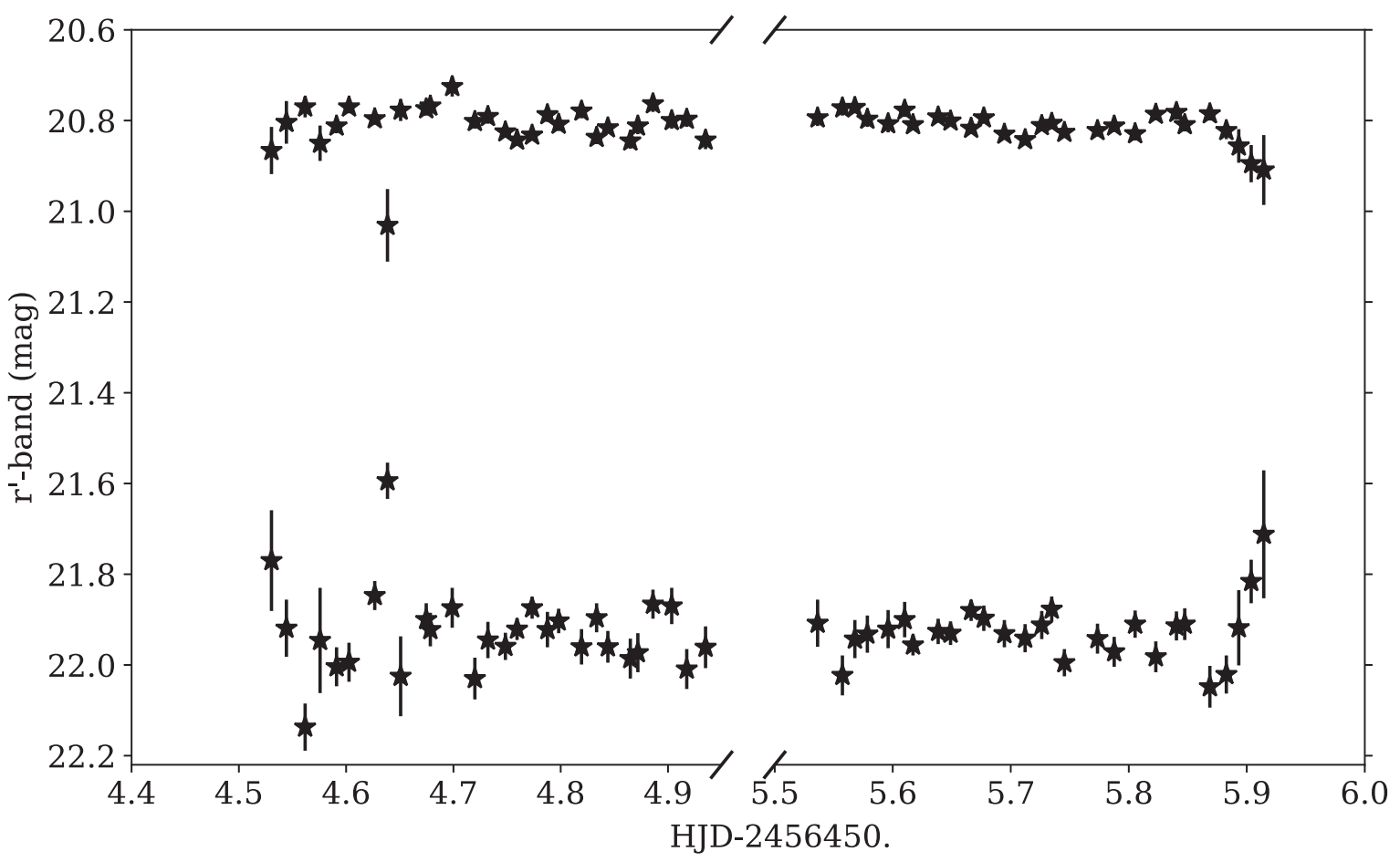

Figure 4. DECam $r^{\prime}$-band light curve of CX1004 (top) and N-E field star (bottom). Note that the first, eight, and final data points in both light curves are affected by correlated variability.

band magnitude measured in Section 2. With this constraint we can set lower limits to the absolute $I$-band magnitude of the accreting binary as a function of its distance. To do this, we first calculate $\mathrm{A}_{\mathrm{I}}$ as a function of the distance utilizing $\mathrm{A}_{\mathrm{I}} / \mathrm{A}_{\mathrm{K}_{\mathrm{s}}}=7.26, A_{\mathrm{K}_{\mathrm{s}}} / \mathrm{E}(\mathrm{J}-$ $\left.\mathrm{K}_{s}\right)=0.528$, and the three-dimensional $\mathrm{E}\left(\mathrm{J}-\mathrm{K}_{s}\right)$ map in the direction of CX1004. In Fig. 5 the solid line shows the resulting absolute I-band magnitude $\mathrm{M}_{\mathrm{I}}$ of the X-ray binary as a function of the distance. $M_{I}$ also represents a lower limit to the absolute magnitude of the donor star since it does not account for the accretion disc contribution to the total light or possible contamination from the field star 1".3 N-E of CX1004. The horizontal lines in the figure mark the I-band absolute magnitude for M-dwarfs (Bessell 1991) and for A to K dwarfs (with subclasses 0, 2, and 5 for each spectral type; Cox 2000). We also show with a dashed line in Fig. 5 the X-ray luminosity $L_{\mathrm{X}}$ as a function of distance. To estimate $L_{\mathrm{X}}$ we used PIMMS to convert the three $0.3-8 \mathrm{keV}$ Chandra-detected X-ray counts of CX1004 to the unabsorbed $0.5-10 \mathrm{keV}$ flux. In this calculation we assumed an absorbed power-law spectrum with index 2.1, which is typical for BH LMXBs in quiescence (Plotkin, Gallo \& Jonker 2013) The absorbing hydrogen column density $N_{\mathrm{H}}$ was obtained using the empirical law $N_{\mathrm{H}} \approx 2.21 \times 10^{21} A_{\mathrm{V}}$ (Güver \& Özel 2009) and $A_{\mathrm{V}} / A_{\mathrm{I}}=1.85$. Note that this calculation of $L_{\mathrm{x}}$ is subject to a large margin for uncertainty given the statistical noise in the low-count detection of the source.

\section{DISCUSSION}

We have shown that the M2/3-type star that is astrometrically consistent with the position of CX1004 and the $\mathrm{H} \alpha$ emission line source is at a distance of $1.3-1.8 \mathrm{kpc}$. If the actual X-ray binary was at the same distance (whether in a hierarchical triple system with this star or not), its donor will have to be of lower mass than an M4 dwarf to contribute $\lesssim 10$ per cent to the $I$-band flux. Given this constraint on the spectral type of the donor, we can estimate the orbital period $\left(\mathrm{P}_{\text {orb }}\right)$ of the accreting binary by employing the relation between the donor star mean density $(\bar{\rho})$ and the orbital period for a Roche lobe filling star: $\mathrm{P}_{\text {orb }}(\mathrm{h}) \approx \sqrt{110 / \bar{\rho}\left(\mathrm{g} \mathrm{cm}^{-3}\right)}$ (Frank, King \& Raine 2002). Using the empirical equations for the mass and radius of low-mass stars by Mann et al. (2016) we derive $\bar{\rho} \approx 21 \mathrm{~g} \mathrm{~cm}^{-3}$ for an M4 dwarf, thus $\mathrm{P}_{\text {orb }} \lesssim 2.3 \mathrm{~h}$. In the case that the X-ray binary is not located at the same distance as the M-dwarf, this cannot be much further away from us since the reddening towards the source is such that does not preclude the detection of the $\mathrm{H} \gamma$ emission line in the averaged spectrum. Given that we cannot quantify the exact value of the reddening we show here the magnitude of its effect on the inferred optical apparent magnitudes for two test distances. The three-dimensional reddening map towards CX1004 yields $\mathrm{A}_{\mathrm{V}}$ $\approx 3.7(4.4)$ mag for a distance of 2.5 (3.0) kpc. Taking the upper limit to the source brightness of $I=21.7$, the above extinction already implies $\mathrm{V}=25.4$ (26.1) for a conservatively adopted A0 spectrum. The reddening in the $B$ band $\left(\lambda_{\text {eff }}=4361 \AA\right)$, where the $\mathrm{H} \gamma$ emission line is still distinguishable, will be even larger. If the X-ray binary is more distant, the combined effect of the larger reddening and distance make it quickly impossible to have detected the $\mathrm{H} \gamma$ and $\mathrm{H} \beta$ emission lines. The above evidence for moderate reddening implies that the X-ray binary has to lie in front of the Galactic bulge and supports a late-type dwarf mass donor (Fig. 5). We also find it likely that the binary is accreting at a low rate given the lack of evidence for HeII $\lambda 4686$ emission in the spectra and the $\mathrm{X}$-ray luminosities estimated for distances $<4 \mathrm{kpc}$ (Fig. 5). These are consistent with the $0.5-10 \mathrm{keV} \mathrm{X}$-ray luminosities observed for both quiescent $\lesssim 4 \mathrm{~h}$ orbital period LMXBs $\left(\approx 10^{30-32} \mathrm{erg} \mathrm{s}^{-1}\right.$; e.g. fig. 3 in Armas Padilla et al. 2014) and non-magnetic CVs $\left(\approx 10^{29-32} \mathrm{erg} \mathrm{s}^{-1}\right.$; Reis et al. 2013). If CX1004 were a CV, the absence of evidence for dwarf nova outbursts in our light curves implies that either we missed them or the source has a lower duty 


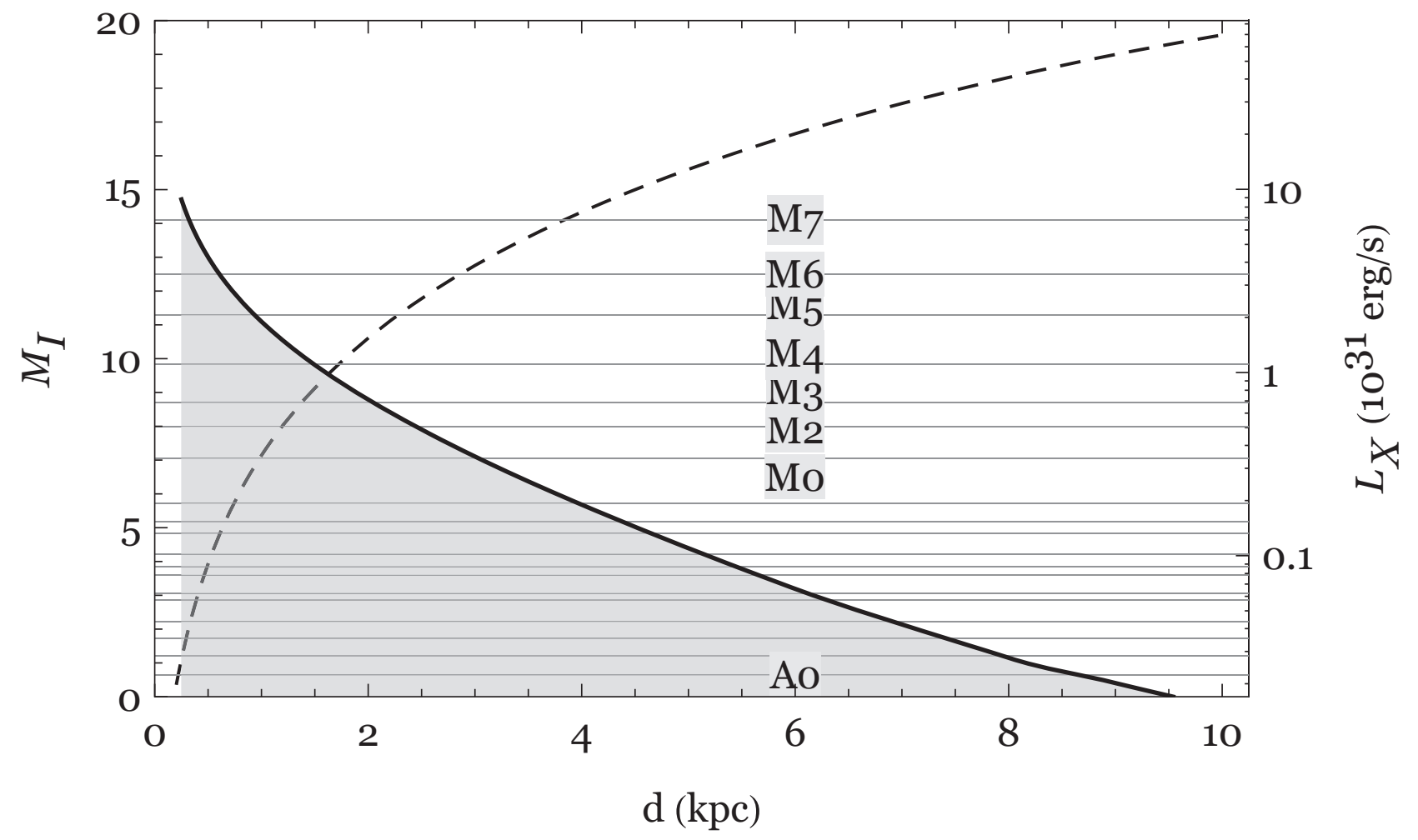

Figure 5. Lower limit for the $I$-band absolute magnitude of the putative donor star in CX1004 (solid line; left vertical scale) and expected X-ray luminosity as a function of distance (dashed line; right vertical scale). The area below the solid line is excluded as the object would be brighter in the $I$ band than observed. The grey horizontal lines mark the absolute magnitudes of M-type dwarfs and of K- to A-type dwarfs in three subclasses: 0,2 , and 5 .

cycle and lower X-ray luminosity compared to non-magnetic CVs that show frequent outburst activity (Britt et al. 2015). In the latter case CX1004 would most likely belong to the WZ Sge class of $\mathrm{CV}$ s which is characterized for having orbital periods $\lesssim 1.4 \mathrm{~h}$ and median outburst recurrence times of 12 yr (Kato 2015).

Another indirect way of characterizing the accreting binary in CX1004 is by analysing the $\mathrm{H} \alpha$ emission line properties. By comparing its FWHM to those measured in a number of selected low accretion rate CVs and LMXBs, Torres et al. (2014) suggested that the $\mathrm{H} \alpha$ line broadening is consistent with CX1004 being either an eclipsing CV or a high-inclination LMXB. Recently, Casares (2018) has presented a comprehensive study on the $\mathrm{H} \alpha$ emission line properties for CVs and quiescent LMXBs. This study shows that only eclipsing $\mathrm{CVs}$ with very short $(\lesssim 2.2 \mathrm{~h})$ orbital periods can have $\mathrm{H} \alpha$ emission line $\mathrm{FWHM} \geq 2200 \mathrm{~km} \mathrm{~s}^{-1}$. As shown in Section 3, the average $\mathrm{H} \alpha$ emission line FWHM in CX1004 exceeds this limit. Thus the accreting binary would either be (i) an eclipsing short orbital period $\mathrm{CV}$, (ii) a high-inclination short orbital period neutron star LMXB, or (iii) a BH LMXB observed at moderate orbital inclination. At this point, we cannot discriminate between the CV and LMXB interpretation. For the CV case, we constrain the donor star to white dwarf mass ratio $(q)$ to be $<0.22$ for $\mathrm{P}_{\text {orb }}<2.2 \mathrm{~h}$ using the empirical exponential relation between $q$ and $\mathrm{P}_{\text {orb }}$ reported in Casares (2018). For the $\mathrm{BH}$ case, limits on the donor star to $\mathrm{BH}$ mass ratio $q$ can be set using the correlation established for quiescent BH LMXBs between this quantity and the $\mathrm{H} \alpha$ double-peak separation (DP) to FWHM ratio: $\log q=$ -23.2log (DP/FWHM) - 6.88 (Casares 2016). Following Casares (2016), we calculated the FWHM and DP by fitting the averaged $\mathrm{X}$-shooter and GMOS $\mathrm{H} \alpha$ profiles with single and 2-Gaussian models, with the latter having Gaussian components with identical width and height. The resulting FWHM and DP values are reported in Table 3. The mass ratio was evaluated through Monte Carlo randomization where DP/FWHM was treated as being normally distributed about its measured value with standard deviation equal to its uncertainty. From the X-shooter and GMOS data we constrain $q$ to $0.09_{-0.02}^{+0.03}$ and $0.08_{-0.03}^{+0.06}$, respectively. The quoted uncertainties correspond to 68 per cent confidence level regions. For an LMXB hosting a $10(5) \mathrm{M}_{\odot} \mathrm{BH}$, the lower limit for $q$ implies a $0.5(0.25)$ $\mathrm{M}_{\odot}$ donor star. This result implies that the possibility of CX1004 being a triple system containing an inner $\mathrm{BH}$ binary accreting from a donor of spectral type later than $\mathrm{M} 4\left(0.26 \mathrm{M}_{\odot}\right)$ is restricted since it requires a $<5 \mathrm{M}_{\odot} \mathrm{BH}$ and these seem to be rare (Casares \& Jonker 2014).

Finally, we derive the RV semi-amplitude of the donor star $\left(\mathrm{K}_{\mathrm{d}}\right)$ using the $\mathrm{H} \alpha \mathrm{FWHM}-\mathrm{K}_{\mathrm{d}}$ correlation for quiescent $\mathrm{CV}$ s and $\mathrm{BH}$ LMXBs (Casares 2015). $\mathrm{K}_{\mathrm{d}}$ is better evaluated when accounting for time variability of the line profile. Therefore, we use for the FWHM the value of $2350 \mathrm{~km} \mathrm{~s}^{-1}$ and r.m.s. $=140 \mathrm{~km} \mathrm{~s}^{-1}$ as measured from the GMOS data (Section 4.2). We obtain $\mathrm{K}_{\mathrm{d}}$ to be $400 \pm 40 \mathrm{~km} \mathrm{~s}^{-1}$ (CV scenario) and $550 \pm 40 \mathrm{~km} \mathrm{~s}^{-1}$ (BH scenario).

\section{CONCLUSIONS}

The photometric data and the broad double-peaked $\mathrm{H} \alpha$ emission line present in its spectrum allow CX1004 to be a $\lesssim 2.2 \mathrm{~h}$ orbital period eclipsing CV or an LMXB. However, neither RV variations nor line broadening is detected in the photospheric lines of the M2/3 dwarf optical counterpart. This implies that the M2/3-type star is 
not the donor star of the accreting binary since radial variations should have been measurable with our observations given the expected RV semi-amplitude $\mathrm{K}_{\mathrm{d}}\left(\simeq 400-550 \mathrm{~km} \mathrm{~s}^{-1}\right)$. The available spectroscopic and photometric data do not allow us to determine whether the accretor is a white dwarf, neutron star, or a $\mathrm{BH}$ or to discriminate between the two possible interpretations for the M2/3 dwarf: an interloper along the line of sight to the accreting binary or the outer companion in a triple system. We note that two candidate hierarchical triple systems with red dwarf outer companions have been reported in the literature: the $3.5 \mathrm{~h}$ orbital period CV RR Pic (Vogt et al. 2017) and the LMXB MAXI J1957+032 (Ravi 2017). The best strategy to constrain the compact object nature in CX1004 is to obtain the orbital period through an RV study of the $\mathrm{H} \alpha$ emission line. For this purpose higher SNR, higher time resolution, and continuous coverage of the potential short orbital period are needed. In addition, RVs measured from the photospheric lines will serve to further test the interloper scenario for the M2/3 star.

\section{ACKNOWLEDGEMENTS}

Based on observations made with ESO Telescopes at the La Silla Paranal Observatory under programme ID 088.D-0096(A) and 095.D-0973(A). This research has used the SIMBAD data base, operated at CDS, Strasbourg, France, and of the NASA's Astrophysics Data System. IRAF is distributed by the National Optical Astronomy Observatory, which is operated by the Association of Universities for Research in Astronomy (AURA) under a cooperative agreement with the National Science Foundation. Tom Marsh is thanked for developing and sharing his package MOLLY. We thank the anonymous referee for constructive comments. MAPT and JC acknowledge support by the Spanish Ministry of Economy, Industry, and Competitiveness (MINECO) under grant AYA201783216-P. MAPT also acknowledges support via a Ramón y Cajal Fellowship (RYC-2015-17854). The work of SR was supported by the Netherlands Research School for Astronomy (NOVA). PGJ and ZKR acknowledge support from European Research Council Consolidator grant 647208. RIH and CBJ acknowledge support from NASA through Chandra Award Number AR3-14002X issued by the Chandra X-ray Observatory Center, which is operated by the Smithsonian Astrophysical Observatory for and on behalf of the National Aeronautics Space Administration under contract NAS8-03060. The work of LW has been supported by the Polish National Science Centre grant no. DEC-2011/03/B/ST9/02573. The OGLE project has received funding from the National Science Centre, Poland, grant MAESTRO 2014/14/A/ST9/00121 and 2015/18/M/ST9/00544 to Andrzej Udalski. COH is supported by NSERC Discovery grant RGPIN-2016-04602, and a Discovery Accelerator Supplement. SR is thankful to Hagai Perets for his insights on the triple scenario.

\section{REFERENCES}

Appenzeller I. et al., 1998, The Messenger, 94, 1

Armas Padilla M., Wijnands R., Degenaar N., Muñoz-Darias T., Casares J., Fender R. P., 2014, MNRAS, 444, 902

Belczynski K., Taam R. E., 2004, ApJ, 603, 690

Belczynski K., Wiktorowicz G., Fryer C. L., Holz D. E., Kalogera V., 2012, ApJ, 757, 91

Bessell M. S., 1991, AJ, 101, 662

Britt C. T. et al., 2013, ApJ, 769, 120

Britt C. T. et al., 2014, ApJS, 214, 10
Britt C. T. et al., 2015, MNRAS, 448, 3455

Carpenter J. M., 2001, AJ, 121, 2851

Casares J., 2015, ApJ, 808, 80

Casares J., 2016, ApJ, 822, 99

Casares J., 2018, MNRAS, 473, 5195

Casares J., Jonker P. G., 2014, Space Sci. Rev., 183, 223

Corral-Santana J. M., Casares J., Muñoz-Darias T., Bauer F. E., MartínezPais I. G., Russell D. M., 2016, A\&A, 587, A61

Cox A. N., 2000, Allen's Astrophysical Quantities. New York, AIP Press, Springer

Damineli A., Almeida L. A., Blum R. D., Damineli D. S. C., Navarete F., Rubinho M. S., Teodoro M., 2016, MNRAS, 463, 2653

DePoy D. L. et al., 2008, in McLean I. S., Casali M. M., eds, Proc. SPIE Conf. Ser., Vol. 7014. Ground-based and Airborne Instrumentation for Astronomy II. SPIE, Bellingham, 70140E

Frank J., King A., Raine D. J., 2002, Accretion Power in Astrophysics. 3rd edn., Cambridge, UK, Cambridge University Press, p. 398

Fryer C. L., Kalogera V., 2001, ApJ, 554, 548

Fryer C. L., Belczynski K., Wiktorowicz G., Dominik M., Kalogera V., Holz D. E., 2012, ApJ, 749, 91

González-Fernández C. et al., 2018, MNRAS, 474, 5459

Gray D. F., 1992, The Observation and Analysis of Stellar Photospheres. Camb. Astrophys. Ser., p. 20

Greiss S. et al., 2014, MNRAS, 438, 2839

Güver T., Özel F., 2009, MNRAS, 400, 2050

Hynes R. I. et al., 2012, ApJ, 761, 162

Johnson C. B. et al., 2017, MNRAS, 466, 129

Jones J. E., Alloin D. M., Jones B. J. T., 1984, ApJ, 283, 457

Jonker P. G. et al., 2011, ApJS, 194, 18

Jonker P. G. et al., 2014, ApJS, 210, 18

Jonker P. G., Nelemans G., 2004, MNRAS, 354, 355

Kalogera V., Webbink R. F., 1998, ApJ, 493, 351

Kato T., 2015, PASJ, 67, 108

Kiel P. D., Hurley J. R., 2006, MNRAS, 369, 1152

Maccarone T. J. et al., 2012, MNRAS, 426, 3057

Mann A. W., Feiden G. A., Gaidos E., Boyajian T., von Braun K., 2016, ApJ, 819, 87

Marsh T. R., Horne K., Shipman H. L., 1987, MNRAS, 225, 551

Marsh T. R., Robinson E. L., Wood J. H., 1994, MNRAS, 266, 137

Miller-Jones J. C. A., 2014, Publ. Astron. Soc. Aust., 31, e016

Minniti D. et al., 2010, New Astron., 15, 433

Modigliani A. et al., 2010, in Silva D. R., Peck A. B., Soifer B. T., eds, Proc. SPIE Conf. Ser., Vol. 7737. Observatory Operations: Strategies, Processes, and Systems III. SPIE, Bellingham, p. 28

Nataf D. M. et al., 2016, MNRAS, 456, 2692

Nidever D. L., Marcy G. W., Butler R. P., Fischer D. A., Vogt S. S., 2002, ApJS, 141, 503

Nishiyama S., Tamura M., Hatano H., Kato D., Tanabé T., Sugitani K., Nagata T., 2009, ApJ, 696, 1407

Osterbrock D. E., Fulbright J. P., Martel A. R., Keane M. J., Trager S. C., Basri G., 1996, PASP, 108, 277

Pfahl E., Rappaport S., Podsiadlowski P., 2003, ApJ, 597, 1036

Plotkin R. M., Gallo E., Jonker P. G., 2013, ApJ, 773, 59

Portegies Zwart S. F., Verbunt F., Ergma E., 1997, A\&A, 321, 207

Ratti E. M. et al., 2013, MNRAS, 428, 3543

Ravi V., 2017, ApJ, 851, 114

Reis R. C., Wheatley P. J., Gänsicke B. T., Osborne J. P., 2013, MNRAS, 430, 1994

Repetto S., Nelemans G., 2015, MNRAS, 453, 3341

Repetto S., Igoshev A. P., Nelemans G., 2017, MNRAS, 467, 298

Romani R. W., 1992, ApJ, 399, 621

Schultheis M. et al., 2014, A\&A, 566, A120

Steeghs D., Jonker P. G., 2007, ApJ, 669, L85

Tonry J., Davis M., 1979, AJ, 84, 1511

Torres M. A. P. et al., 2014, MNRAS, 440, 365

Torres M. A. P., Jonker P. G., Miller-Jones J. C. A., Steeghs D., Repetto S., Wu J., 2015, MNRAS, 450, 4292

Udalski A. et al., 2012, Acta Astron., 62, 133 
Udalski A., Szymański M. K., Szymański G., 2015, Acta Astron., 65, 1

Ugliano M., Janka H.-T., Marek A., Arcones A., 2012, ApJ, 757, 69

VernetJ. et al., 2011, A\&A, 536, A105

Vogt N., Schreiber M. R., Hambsch F.-J., Retamales G., Tappert C., Schmidtobreick L., Fuentes-Morales I., 2017, PASP, 129, 014201

Wevers T. et al., 2016a, MNRAS, 458, 4530

Wevers T. et al., 2016b, MNRAS, 462, L106
Wevers T. et al., 2017, MNRAS, 466, 163

Wu J. et al., 2015, MNRAS, 448, 1900

Yungelson L. R., Lasota J.-P., Nelemans G., Dubus G., van den Heuvel E. P. J., Dewi J., Portegies Zwart S., 2006, A\&A, 454, 559

Zhong J. et al., 2015, AJ, 150, 42

This paper has been typeset from a $\mathrm{T}_{\mathrm{E}} \mathrm{X} / \mathrm{LT} \mathrm{E} \mathrm{X}$ file prepared by the author. 